\title{
COMPOSITOS POLÍMERO-MADEIRA PREPARADOS POR POLIMERIZAÇÃO in situ COM MMA EM PROPRIEDADES FÍSICAS DE Pinus elliottii
}

\author{
POLYMER-WOOD COMPOSITES PREPARED BY POLYMERIZATION in SitU WITH MMA IN \\ PHYSICAL PROPERTIES PinUs elliottii
}

\author{
Andrey Pereira Acosta ${ }^{1}$, Henrique Römer Schulz², Ezequiel Gallio ${ }^{3}$, Kelvin Techera Barbosa ${ }^{4}$, \\ Darci Alberto Gatto ${ }^{5}$
}

1, 2, 3, 4, 5 Universidade Federal de Pelotas, Pelotas, Rio Grande do Sul, Brasil - andreysvp@gmail.com, henriqueschulz09@hotmail.com, kelvintecherabarbosa@gmail.com, egeng.florestal@gmail.com \& darcigatto@yahoo.com

\section{RESUMO}

Este estudo tem como objetivo, a avaliação de propriedades físicas de Pinus elliottii polimerizada in situ com metacrilato de metila. Para isso, foram confeccionados corpos de prova de $25 \mathrm{~mm} \times 25 \mathrm{~mm} \times 50 \mathrm{~mm}$. As análises foram conduzidas por meio das técnicas normativas da ASTM - American society for testing and materials D 143-94 (ASTM, 2014) e D 2395 (ASTM, 2017). As madeiras tratadas com metacrilato de metila apresentaram valores médios inferiores de massa específica básica e valores superiores relacionados a questões de retratibilidade (inchamento e contração), quando comparadas ao grupo controle, sendo significativas tais diferenças para determinadas propriedades, tais como, massa específica básica, contração e inchamento linear máxima no plano tangencial. Determinados parâmetros físicos, como inchamento, contração, massa específica aparente e estabilidade dimensional da madeira apresentam características de serem diretamente ou inversamente proporcional, conforme constatado na correlação de Pearson. Nesse contexto, constatou-se modificações nas propriedades físicas analisadas, com incremento de $79,05 \%$ na massa especifica aparente e uma subtração para a contração e inchamento proporcionando a madeira de Pinus elliottii uma otimização em sua estabilidade dimensional.

PALAVRAS-CHAVE: Anisotropia, Estabilidade dimensional, Inchamento, Tratamento da madeira.

\begin{abstract}
This study aims to evaluate the physical properties of Pinus elliottii polymerized in situ with methyl methacrylate. For this, $25 \mathrm{~mm} \times 25 \mathrm{~mm} \times 50 \mathrm{~mm}$ specimens were made. The analyzes were conducted using the normative techniques of ASTM - American Society for Testing and Materials D 143-94 (ASTM, 2014) and D 2395 (ASTM, 2017). Woods treated with methyl methacrylate presented lower average values of basic specific gravity and higher values related to shrinkage (swelling and contraction) when compared to the control group, being significant such differences for certain properties, such as basic specific mass, contraction and maximum linear swelling in the tangential plane. Certain physical parameters, such as swelling, contraction, apparent specific mass and dimensional stability of the wood have characteristics of being directly or inversely proportional, as found in the Pearson correlation. In this context, it was found changes in the physical properties analyzed, with an increase of $79.05 \%$ in apparent specific mass and a subtraction for contraction and swelling, providing the Pinus elliottii wood an optimization in its dimensional stability.
\end{abstract}

KEY WORDS: Anisotropy, Dimensional stability, Swelling, Wood treatment. 


\section{INTRODUÇÃO}

A madeira é um material que pode apresentar limitações quando utilizada de forma isolada, em função de finalidade, necessitando de tratamentos que modifiquem positivamente suas propriedades. Nesse contexto, existe um grande interesse na execução de pesquisas que visem o desenvolvimento de novas técnicas e métodos visando ampliar a possibilidade de uso e a durabilidade da madeira (MENEZES et al., 2014).

Essas melhorias podem ser adquiridas por meio da obtenção de materiais compósitos, os quais são formados pela combinação de dois ou mais materiais de características distintas (MATTOS et al., 2015a). Os compósitos polímeros madeira obtidos pela impregnação, e posterior polimerização de monômeros no interior dos poros, constituem-se em uma das alternativas viáveis a serem utilizadas visando a melhoria das propriedades físicas e mecânicas do material formado (HADI et al., 2013).

Um dos monômeros que é utilizado para a polimerização é o metacrilato de metila (MMA), o qual é um produto químico orgânico e de constituição definida. $O$ interesse da utilização de MMA na confecção de compósitos polímero madeira dá-se devido ao seu baixo custo, fácil síntese, estabilidade química, translucidez, e pela facilidade de catálise (MATTOS et al., 2015b), podendo ser aplicado como intermediário em sínteses orgânicas diversas.

Entretanto, a impregnação de substâncias químicas (monômeros) ocorrem eficientemente se estas possuem baixo peso molecular e baixa viscosidade, facilitando assim seu fluxo entre os poros e capilares, efetuando após o processo de polimerização in situ modificações na estrutura química da madeira (DING et al., 2008; DING et al., 2013; ISLAM et al., 2014).

Visando um tratamento de polimerização in situ eficiente, é necessário que o material apresente elevada porosidade e baixa massa específica (DING et al., 2013; MATTOS et al., 2015c). A madeira de Pinus elliottii caracteriza-se por apresentar essas propriedades. Entretanto, algumas espécies desse gênero possuem limitações quanto ao emprego no setor madeireiro por apresentarem baixa estabilidade dimensional (TRIANOSKI et al., 2013), necessitando assim de tratamentos que visem a melhora dessas propriedades.

Nesse contexto, o objetivo desse trabalho é a avaliação das alterações de propriedades físicas da madeira de Pinus elliottii polimerizada in situ com metacrilato de metila.

\section{MATERIAL E MÉTODOS}

\section{Aquisição da matéria prima}

Para o presente estudo foram utilizadas madeiras da espécie Pinus elliottii (aproximadamente 15 anos de idade, sendo considerada madeira jovem). Confeccionaram-se 10 corpos de prova com dimensões de $25 \mathrm{~mm} \times 25 \mathrm{~mm} \times 50$ $\mathrm{mm}$ na direção (radial, tangencial e longitudinal), visando a realização dos testes físicos.

Posteriormente, encaminharam-se os mesmos a uma sala climatizada (temperatura de $20 \pm 2^{\circ} \mathrm{C}$ e $65 \pm 3 \%$ de umidade relativa) até estabilização da massa, obtendo-se assim o teor de umidade de equilíbrio higroscópico de $12 \%$.

\section{Tratamento com MMA}

Para o tratamento utilizou-se uma solução contendo Metacrilato de metila 99\% FG (densidade: 0,936g/mol a $25^{\circ} \mathrm{C}$ ) e peróxido de benzoila na proporção de $1,5 \%$ em massa como acelerador de polimerização.

As amostras tratadas antes do processo de impregnação foram encaminhadas a uma estufa laboratorial com cerca de $50^{\circ} \mathrm{C}$ por um período de 5 dias, com objetivo de remoção da água química presente na parede celular da madeira, atingindo o valor teórico de $0 \%$ de umidade.

Para a impregnação utilizou-se uma autoclave horizontal de pequeno porte (capacidade de 2,3 L), aonde foi preenchida em seu volume com corpos de prova, em seguida submetidos a um vácuo inicial de 40 minutos, com o auxílio de uma bomba de vácuo $(600 \mathrm{mmHg})$, método denominado como célula cheia, onde é retirada todo o ar presente na madeira facilitando assim a impregnação.

Em seguida foi inserida a solução de MMA por conta da diferença de pressão do ambiente externo com o interior da autoclave. Por sequência aplicou-se com o uso de compressor uma pressão de $8 \mathrm{kgf.cm}^{-2}$ durante 180 minutos, após deixado escorrer o excesso da solução por um período de 10 minutos.

Os corpos de prova, após o processo de impregnação, foram encaminhados a uma estufa laboratorial, regulada com temperatura constante de $50^{\circ} \mathrm{C}$ por um período de 24 horas e em seguida elevada a temperatura para $70^{\circ} \mathrm{C}$ por um período de 72 horas visando a finalização do processo de polimerização in situ do MMA no interior da madeira.

\section{Análise de propriedades físicas}

A caracterização das propriedades físicas da madeira 
tratada foi realizada através de adaptações das normas American Society for Testing and Materials D 143-94 (ASTM, 2014) e ASTM D 2395 (2017).

A massa específica aparente ao teor de umidade de $12 \%$ foi determinada por meio da Equação 1. Para tanto, obtiveram-se as massas com uma balança analítica (precisão de $\pm 0,01 \mathrm{~g}$ ), enquanto a aquisição do volume (pelo método estereométrico) foi feita com o uso de paquímetro digital (precisão de $\pm 0,001 \mathrm{~mm}$ ).

$$
\rho_{12 \%}=\frac{M_{12 \%}}{V_{12 \%}}
$$

Em que: $\rho_{12} \%=$ massa específica aparente ao teor de umidade de $12 \%\left(\mathrm{~g} / \mathrm{cm}^{3}\right) ; \mathrm{M}_{12} \%$ = massa da amostra ao teor de umidade de $12 \%$ (g); e $\mathrm{V}_{12} \%$ = volume da amostra ao teor de umidade de $12 \%\left(\mathrm{~cm}^{3}\right)$.

Analisou-se a influência do tratamento com MMA na contração e no inchamento da madeira nos planos anatômicos tangencial e radial. Para isso, as amostras foram imersas em água até completa saturação. Após saturadas (verificada pela estabilização da massa), todas amostras foram encaminhadas a uma estufa laboratorial, ajustada à $103 \pm 2{ }^{\circ} \mathrm{C}$, visando a obtenção da massa seca.

Isso possibilitou, com a auxílio das Equações 2 a 5, a determinação da contração e inchamento no plano tangencial (Equações 2 e 4) e do inchamento nos respectivos planos anatômicos (Equações 3 e 5). A obtenção das dimensões foi feita com o uso de paquímetro digital, com precisão de $\pm 0,001 \mathrm{~mm}$.

$$
\begin{aligned}
& \beta_{\mathrm{T}}=\left(\frac{\mathrm{T}_{\mathrm{S}}-\mathrm{T}_{0}}{\mathrm{~T}_{\mathrm{S}}}\right) * 100 \\
& \beta_{\mathrm{R}}=\left(\frac{\mathrm{R}_{\mathrm{S}}-\mathrm{R}_{0}}{\mathrm{R}_{\mathrm{S}}}\right) * 100 \\
& \alpha_{\mathrm{T}}=\left(\frac{\mathrm{T}_{\mathrm{S}}-\mathrm{T}_{0}}{\mathrm{~T}_{0}}\right) * 100 \\
& \alpha_{\mathrm{R}}=\left(\frac{\mathrm{R}_{\mathrm{S}}-\mathrm{R}_{0}}{\mathrm{R}_{0}}\right) * 100
\end{aligned}
$$

Em que: $\beta_{T}=$ contração linear máxima no plano anatômico tangencial (\%); $\beta_{R}=$ contração linear máxima no plano anatômico radial (\%); $\alpha_{T}=$ inchamento linear máximo no plano anatômico tangencial (\%); $\alpha_{R}=$ inchamento linear máximo no plano anatômico radial (\%); $T_{s}$ e Rs = dimensões dos planos tangencial e radial em condição saturada de umidade $(\mathrm{mm}) ;$ e $\mathrm{T}_{0}$ e $\mathrm{R}_{0}=$ dimensões dos planos tangencial e radial em condição seca à $103 \pm 2{ }^{\circ} \mathrm{C}(\mathrm{mm})$.
Verificou-se o coeficiente de anisotropia (CA) nos planos radiais e tangenciais para todos os tratamentos, com base nos valores obtidos na contração e inchamento lineares máximos, utilizando as equações 6 e 7.

$$
\begin{aligned}
\mathrm{CA}_{\beta} & =\frac{\beta_{\mathrm{T}}}{\beta_{\mathrm{R}}} \\
\mathrm{CA}_{\alpha} & =\frac{\alpha_{\mathrm{T}}}{\alpha_{\mathrm{R}}}
\end{aligned}
$$

Em que: $\mathrm{CA}_{\beta}=$ coeficiente de anisotropia para contração; $\mathrm{CA}_{\alpha}=$ coeficiente de anisotropia para inchamento; $\beta_{\mathrm{T}}=$ contração linear máxima no plano anatômico tangencial (\%); $\beta_{\mathrm{R}}=$ contração linear máxima no plano anatômico radial (\%); $\alpha_{\mathrm{T}}=$ inchamento linear máximo no plano anatômico tangencial (\%); e $\alpha_{R}=$ inchamento linear máximo no plano anatômico radial (\%).

\section{Análise estatística}

Utilizou-se o delineamento inteiramente casualizado (DIC) com o tratamento com MMA e o tratamento controle. A homogeneidade dos resultados foi avaliada por meio do teste Boxplot, em que estes se mostraram satisfatórios para aplicação dos testes paramétricos. Em caso de rejeição da hipótese nula, foram realizados os testes de Tukey e $\mathrm{F}$ em nível de $5 \%$ de significância de erro. A correlação entre as variáveis de interesse no estudo foi verificada por meio da correlação de Pearson, em 5\% de probabilidade de erro.

\section{RESULTADOS E DISCUSSÃO}

A impregnação da madeira de Pinus elliottii com metil metacrilato (MMA) provocou alterações nas propriedades físicas avaliadas. Isso pode ser notado pelo aumento significativo na massa específica aparente de aproximadamente $79 \%$ (Tabela 1 ).

Esse comportamento também foi observado por Hadi et al. (2013) e Ding et al. (2008), os quais após tratamentos de diferentes espécies madeireiras de densidades semelhantes ao P. elliottii (Jabon e híbrido de Poplar, respectivamente) com MMA, obtiveram incrementos de aproximadamente $35 \%$ e $220 \%$ em comparação as massas específicas iniciais.

O tratamento com MMA causa modificações nas propriedades físicas da madeira devido ao fato desse monômero estar impregnado e polimerizado nos espaços vazios e capilares da madeira, aumentando assim sua massa específica, fazendo com que se ocorra uma maior 
repelência da água e assim uma menor contração e inchamento. Mesmo que esse monômero não interaja de maneira direta com os constituintes químicos principais da madeira (celulose, hemicelulose e lignina), o mesmo é capaz de alterar as propriedades físicas da madeira em função de sua natureza (MATTOS et al., 2015d).

Tabela 1. Resumo estatístico e valores médios para os parâmetros relacionados a massa específica aparente ao teor de umidade de $12 \%\left(\rho_{12} \%\right)$ das madeiras de $P$. elliottii dos grupos controle e tratadas com MMA.

\begin{tabular}{cc}
\hline Tratamento & $\boldsymbol{\rho}_{\mathbf{1 2} \%}\left(\mathbf{g} \cdot \mathbf{c m}^{-3}\right)$ \\
\hline Controle & $0,377( \pm 0,134) \mathrm{b}$ \\
MMA & $0,675( \pm 0,023) \mathrm{a}$ \\
CV & $34,08 \%$ \\
DP & $\pm 0,179 \mathrm{~g} . \mathrm{cm}^{-3}$ \\
F & $48,20 *$ \\
\hline
\end{tabular}

Em que: valores entre parênteses e sobescritos representam 0 desvio padrão da média do tratamento e letras diferentes, nas colunas, indicam diferenças significativas conforme o teste LSD Fisher, em 5\% de probabilidade de erro; CV = coeficiente de variação entre os tratamentos; DP = desvio padrão entre os tratamentos; $\mathrm{e}^{*}=$ apresenta diferença significativa pelo teste $\mathrm{F}$ $(p<0,05)$.

De maneira geral, a polimerização com MMA causou alteração significativa somente para a contração $(\beta)$ no plano tangencial (Figura 1).

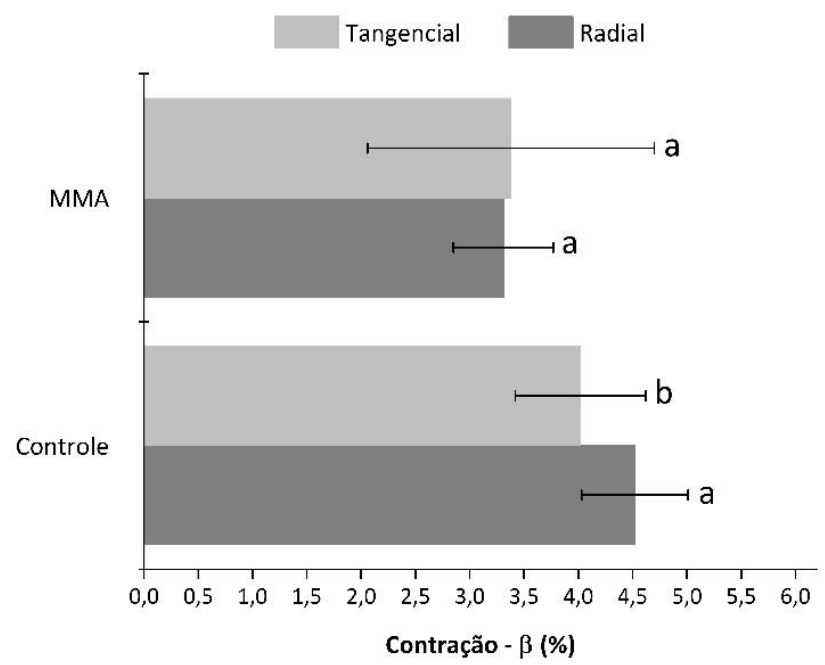

Figura 1. Contração média da madeira de $P$. elliottii tratada com MMA. Em que: letras iguais, em um mesmo plano anatômico, não apresentam diferença significativa em $5 \%$ de probabilidade de erro $(p<$ 0,05 ) pelos testes $F$ e de Tukey, e as linhas horizontais apresentam o desvio padrão da média.
As reduções das amostras tratadas quando comparadas aos tratamentos controle foram de 26,79\%, $15,80 \%$ e $6,42 \%$ para os planos tangencial $\left(\beta_{T}\right)$, radial $\left(\beta_{R}\right)$ e anisotropia da contração $\left(C_{\beta}\right)$, respectivamente. Para a anisotropia da contração $\left(A C_{\beta}\right)$, obteve-se uma redução de $6,42 \%$ das madeiras tratadas com MMA $(1,05)$ em comparação com o tratamento controle $(1,12)$.

Este resultado pode estar relacionado ao efeito de preenchimento efetuado pelo polímero (que possuí altas características hidrofóbicas) nos espaços vazios da madeira (Dong et al., 2019).

Quanto ao inchamento $(\alpha)$, nota-se a existência de diferença significativa somente para o plano tangencial, assim como o ocorrido na contração $(\beta)$. Houve redução do percentual de inchamento em ambos os planos anatômicos em função do tratamento com MMA: 30,80\% (no plano tangencial) e 15,99\% (no plano radial).

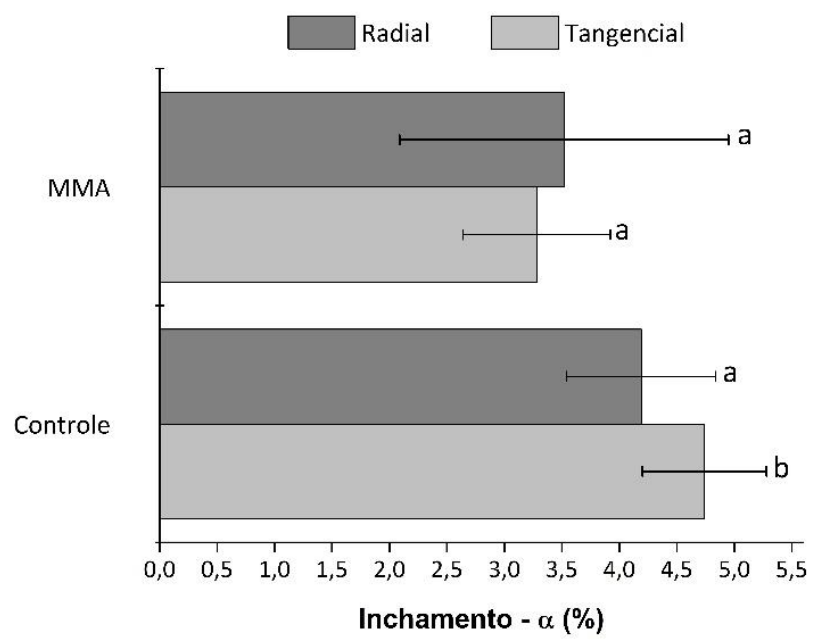

Figura 2. Inchamento médio da madeira de $P$. elliottii tratada com MMA. Em que: letras iguais, em um mesmo plano anatômico, não apresentam diferença significativa em $5 \%$ de probabilidade de erro $(p<0,05)$ pelos testes $F$ e de Tukey, e as linhas horizontais apresentam o desvio padrão da média.

Assim como para a contração, o tratamento com MMA resultou em uma redução de aproximadamente $7 \%$ na anisotropia do inchamento $(A C \alpha)$, aonde a madeira de $P$. elliottii apresentou um AC $\alpha$ de 1,13, e após o tratamento com MMA, de 1,05.

O plano tangencial apresentou um percentual de contração e inchamento superior ao plano radial. Isso é explicado pelas diferentes distribuições e dimensões dos elementos anatômicos que constituem a madeira (raios, canais resiníferos, traqueideos), propiciando variações dimensionais distintas nos diferentes planos anatômicos 
(OLIVEIRA et al., 2010). Nesse contexto, quanto menor for a diferença entre os planos tangencial e radial, mais estáveis dimensionalmente é a madeira. No geral, coeficientes de anisotropia menores que 1,5 qualificam a madeira como excelentes, enquanto valores de anisotropia compreendidos entre 1,5 e 2,0 caracterizam madeiras normais (LOGSDON et al., 2008).

A madeira tratada com metil metacrilato apresenta uma redução nos parâmetros de contração e inchamento, devido a polimerização do monômero no interior do material tratado (ZHANG et al., 2006, DING et al., 2013). Os efeitos nas madeiras tratadas com MMA, são benéficos, sendo diretamente ligados a redução dos parâmetros de contração e inchamento, devido a polimerização do monômero no interior da madeira reduzir a capacidade higroscópica desse material, no que se refere à capacidade de absorver e perder umidade (MEYER, 1977; ISLAM et al., 2014; MATTOS et al., 2015e).

Considerando que o MMA em si apresenta pouca ou nenhuma reatividade com a parede celular da madeira (MATTOS et al., 2015f), sua presença no interior da madeira dificulta a absorção e perda de umidade por parte desse material. Isso devido à madeira ser altamente higroscópica, em função de sua composição química e a presença dos radicais hidroxila $(\mathrm{OH})$, os quais possuem elevada afinidade com a água.

Ainda, XIE et al. (2013) ressaltam que o polímero de metacrilato de metila (PMMA) preenche principalmente a luminária celular, fazendo assim com que a madeira não adquira nem perca umidade com a mesma facilidade da madeira in natura.

O teste de correlação de Pearson demonstra o nível de correlação existente entre as propriedades, aonde valores próximos à 1 ou -1 representam elevada correlação (direta ou inversa, respectivamente), e valores próximos a 0 indicam a ausência de correlação.

Assim, observa-se a existência de correlações diretas e significativas entre: as anisotropias da contração $\left(\mathrm{CA}_{\beta}\right)$ e do inchamento $\left(C A_{\alpha}\right)$; contração tangencial $\left(\beta_{T}\right)$ e contração radial $\left(\beta_{R}\right)$; o inchamento radial $\left(\alpha_{R}\right)$ com a $\beta_{\text {T }}$ e $\beta_{R}$; inchamento no plano tangencial ( $\left.\alpha T\right)$ com o $\alpha_{R}, \beta_{T}$ e $\beta_{R}$ (Tabela 2).

A massa específica aparente $\left(\rho_{12} \%\right)$ demonstrou correlação significativa e inversa com a $\beta_{T}$ e $\alpha_{T}$, enquanto $\beta_{R}$ e $\alpha_{R}$ apresentaram esse tipo de correlação somente com as anisotropias da contração $\mathrm{CA}_{\beta}$ e $\mathrm{CA}_{\alpha}$. Estas, por sua vez, não apresentaram correlação com a $\beta_{T}$, o $\alpha_{T}$ e a $\rho_{12} \%$.
Tabela 2. Correlação de Pearson para as propriedades físicas avaliadas da madeira de $P$. elliottii tratada com MMA.

\begin{tabular}{cccccccc}
\hline & $\mathbf{C A}_{\boldsymbol{\beta}}$ & $\mathbf{C A}_{\boldsymbol{\alpha}}$ & $\boldsymbol{\beta}_{\mathbf{R}}$ & $\boldsymbol{\beta}_{\mathbf{T}}$ & $\boldsymbol{\alpha}_{\mathbf{R}}$ & $\boldsymbol{\alpha}_{\boldsymbol{T}}$ & $\boldsymbol{\rho}_{\mathbf{1 2}}$ \\
\hline $\mathrm{CA}_{\beta}$ & 1 & 1 & $-0,71$ & 0,16 & $-0,70$ & 0,16 & $-0,38$ \\
$\mathrm{CA}_{\alpha}$ & & 1 & $-0,71$ & 0,16 & $-0,71$ & 0,16 & $-0,38$ \\
$\beta_{R}$ & & & 1 & 0,49 & 0,99 & 0,49 & $-0,09$ \\
$\beta_{T}$ & & & & 1 & 0,48 & 1 & $-0,74$ \\
$\alpha_{R}$ & & & & & 1 & 0,48 & $-0,09$ \\
$\alpha_{T}$ & & & & & & 1 & $-0,74$ \\
$\rho_{12 \%}$ & & & & & & & 1 \\
\hline
\end{tabular}

Em que: coeficientes destacados com a cor vermelha apresentam correlação significativa $(p<0,05) ; \mathrm{CA}_{\beta}=$ anisotropia da contração; $C A \alpha=$ anisotropia do inchamento; $\beta_{\mathrm{T}}$ e $\beta_{\mathrm{R}}=$ contração linear máxima (\%) no plano tangencial e radial, respectivamente; $\alpha_{T}$ e $\alpha_{R}=$ inchamento linear máximo (\%) no plano tangencial e radial, respectivamente; e $\rho_{12} \%=$ massa específica aparente ao teor de umidade de $12 \%\left(\mathrm{~g} \cdot \mathrm{cm}^{-3}\right)$.

Essa correlação é atribuida ao incremento na densidade da madeira com o monômero, a madeira tratada reduz sua higrospicidade por conta do preenchimento com o MMA, material que causa a não absorção da água pela parede celular expondo a água ao contato direto com o polímero, sendo assim conforme mais acentuada a densificação da madeira polimerizada in situ, maior será a correlação entre os parâmetros avaliados.

\section{CONCLUSÕES}

Ao se analisar o processo de polimerização in situ com o monômero metacrilato de metila, constata-se uma modificação nas propriedades físicas.

A massa específica aparente demostrou um incremento de $79,05 \%$ com o tratamento com metacrilato de metila, observou-se uma correlação entre o aumento da massa específica com os parâmetros físicos avaliados.

Para a contração e inchamento, destaca-se as alterações para o plano tangencial, este demostrando evidentes reduções, no entanto não se obteve valores significativos para o plano anatômico radial.

Em relação a estabilidade dimensional o tratamento proporcionou redução no parâmetro da anisotropia, tornando assim a madeira de $P$. elliotiii tratada com metacrilato de metila notoriamente mais estável dimensionalmente.

Neste contexto, a modificação com MMA se mostra um promissor tratamento para otimização em propriedades físicas da madeira de $P$. elliottii. 


\section{AGRADECIMENTOS}

O presente trabalho foi realizado com apoio da Coordenação de Aperfeiçoamento de Pessoal de Nível Superior - Brasil (CAPES) - Código de Financiamento 001.

\section{REFERÊNCIAS}

ASTM - AMERICAN SOCIETY FOR TESTING AND MATERIALS. Standard test methods for density and specific gravity (relative density) of wood and wood-based materials. ASTM D 2395- 17. West Conshohocken, 2017.

ASTM - AMERICAN SOCIETY FOR TESTING AND MATERIALS. Standard test methods for small clear specimens of timber. ASTM D 143 - 94. Philadelphia, 2014.

DONG, Y. et al. Various polymeric monomers derived from renewable rosin for the modification of fast-growing poplar wood. Composites Part B: Engineering, v.174, p.106902, 2019.

DING, W.D. et al. Mechanical properties of MMA-hardened hybrid poplar wood. Industrial Crops and Products, v.46, p.304310, 2013.

DING, W.D. et al. Relationship between wood porosity, wood density and methyl methacrylate impregnation rate. Wood Material Science \& Engineering, v.3, n.1-2, p.62-70, 2008.

HADI, Y.S. et al. Physical and mechanical properties of methyl methacrylate impregnated jabon wood. Journal of the Indian Academy of Wood Science, v.10, n.2, p.77-80, 2013.

ISLAM, M.S. et al. The chemical modification of tropical wood polymer composites. Journal of Composite Materials, v.48, n.7, p.783-789, 2014.

LOGSDON, N.B. et al. Caracterização físico-mecânica da madeira de Cedro-marinheiro, Guarea trichilioides L. (Meliaceae). Scientia Forestalis, v.36, n.77, p.43-51, 2008.

MATTOS, B.D. et al. Compósitos polímero-madeira preparados por polimerização in situ: conceitos, parâmetros de processo e propriedades. Ciência da Madeira, v.6, n.3, p.128-148, 2015.

MENEZES, W.M. et al. Modificação térmica nas propriedades físicas da madeira. Ciência Rural, v.44, n.6, p.1019-1024, 2014.

MEYER, J.A. Wood-plastic materials and their current commercial applications. Polymer-Plastics Technology and Engineering, v.9, n.2, p.181-206, 1977.

OLIVEIRA, J.T.S. et al. Avaliação da retratibilidade da madeira de sete espécies de Eucalyptus. Revista Árvore, v.34, n.5, p.929-936, 2010.

TRIANOSKI, R. et al. Avaliação da estabilidade dimensional de espécies de pinus tropicais. Floresta e Ambiente, v.20, n.3, p.398406, 2013.

XIE, Y. Q. et al. Effects of chemical modification on the mechanical properties of wood. Europen Journal Wood, v.71, n.4, p.401-416,
2013.

ZHANG, Y. et al. Effect of impregnation and in-situ polymerization of methacrylates on hardness of sugar maple wood. Journal of Applied Polymer Science, v.99, n.4, p.1674-1683, 2005.

Recebido em 20-06-2019 Aceito em 03-09-2019 